\title{
Highlights from H.E.S.S.
}

\author{
Mathieu de Naurois ${ }^{1, a}$ for the H.E.S.S. collaboration \\ ${ }^{1}$ LLR - IN2P3/CNRS - Ecole Polytechnique
}

\begin{abstract}
In the 15 years since its construction, the H.E.S.S. gamma-ray observatory has allowed the study of the Very High Energy gamma-ray sky at resolutions and sensitivities which were never before possible. During this period H.E.S.S. has discovered a rich zoo of both galactic and extra galactic source classes, made measurements of the galactic cosmic ray spectrum and placed limits on fundamental physical processes.

A summary of the latest H.E.S.S. results for these source classes has been presented at the conference, describing the most interesting new observations and their physical interpretation. Additionally the latest upgrades and improvements to the H.E.S.S. hardware and data analyses, and the future science prospects for the experiment have been described.
\end{abstract}

\section{Introduction}

The H.E.S.S. array, inaugurated 15 years ago, revolutionized our view on the Very High Energy (VHE) gamma-ray sky with more than 100 sources discovered. A second phase of the experiment started in 2012, with the installation of the fifth, largest telescope ( $28 \mathrm{~m}$ diameter), allowing the threshold to be lowered from $\sim 100 \mathrm{GeV}$ down to $\sim 20 \mathrm{GeV}$ and bridge the gap with space instruments such as Fermi-LAT. To date, H.E.S.S. is the only hybrid ${ }^{1}$ in the field, thus paving the way for the next generation Cherenkov Telescope Array. In the last years, tremendous efforts went into the design and optimization of analysis software able to exploit simultaneously monoscopic \& stereoscopic events in a consistent way. At the same time, the field of VHE $\gamma$-ray astronomy is experiencing a kind of phase-transition, where single discoveries tend to have less importance and where population studies and detailed analysis of sources of specific interest are opening new fields. In this context, a new paradigm of simulation (Section 2.3) is now emerging and opening new science capabilities, with unprecedented resolution and understanding of the instrument.

The last years have seen the long awaited emergence of multi-wavelength, multi-messenger astronomy, advocated for since decades, with the discovery of gravitational waves from a binary neutron star merger [1,2] and more recently, with the association of a very high energy neutrino observed by IceCube with an active galactic nuclei [3]. H.E.S.S., thanks to its very fast reaction time and slewing speed, is actively involved in this new time-domain astronomy.

After 15 years of operations, the H.E.S.S.-I cameras have been fully refurbished in 2016 with new electronics, inspired from the design of CTA, to optimize the overlap with CT5 (reduced threshold and dead-time) and ease maintenance. Ongoing negotiations to operate the array beyond 2019 are progressing well, and a wealth of new results are expected in the coming years.

\footnotetext{
a e-mail: denauroi@in2p3.fr

1 i.e. array consisting of telescopes of different size operated together
} 


\section{The H.E.S.S. view of the Milky Way}

The H.E.S.S. collaboration recently released a bundle of 14 publications in a special edition of Astronomy $\mathcal{G}$ Astrophysics [4], focusing on the H.E.S.S.-I analysis of the Galactic Plane. This major release contains in particular:

- the H.E.S.S. Galactic Plane survey [5], cornerstone of the publication and the major project of the collaboration over the last 10 years;

- the first population studies, on Pulsar Wind Nebula (PWN) [6] and Supernova Remnants (SNR) [7];

- detailed analysis on several sources, and in particular first evidence of emission beyond the shell of a SNR [8];

- a comprehensive view of the Galactic Center region, including diffuse emission [9];

- upper limits on several specific objects and/or potential new source classes.

\subsection{H.E.S.S. Galactic Plane Survey}

The H.E.S.S. Galactic plane survey (HGPS) [5] is a major long-term project, corresponding to $\sim 2700$ hours of high-quality observations acquired with the H.E.S.S.-I telescope array from 2004 to 2013. Results have already been published on a small $(\sim 10 \%)$ fraction of the current data set $[10,11]$.

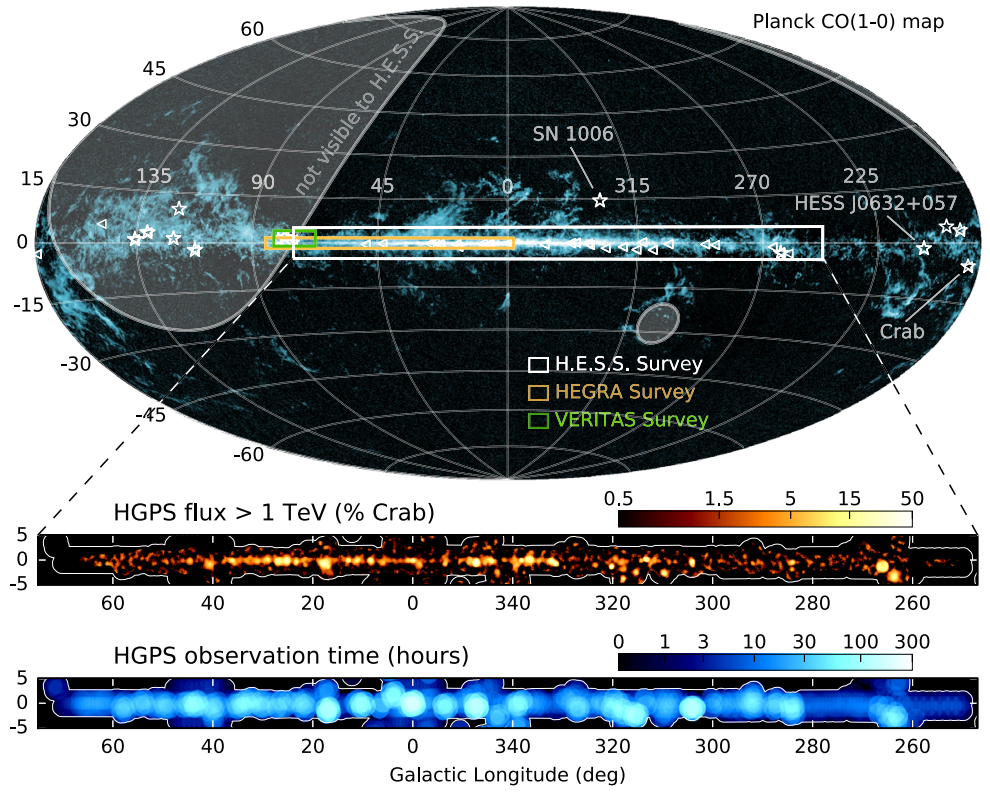

Figure 1. H.E.S.S. Galactic Plane Survey region, flux map and exposure map (from top to bottom). The all-sky image on the top panel shows a Planck CO Map with Fermi-LAT identified Galactic 1FHL sources (triangles) and the 15 known Galactic TeV sources (white stars) outside the HGPS region. The HEGRA Galactic Plane Survey [12] and the VERITAS Cygnus survey [13, 14] regions are illustrated in blue and green, respectively. From [5]. 
The region of the Milky Way covered by the HGPS (Galactic longitude between -110 and 65 degrees and Galactic latitude $|b|<3.5$ degrees) is depicted as a white rectangle in Fig. 1 and compared to the HEGRA (in blue) and VERITAS Cygnus (in green) surveys.

A catalogue of 77 cosmic accelerators was derived from the HGPS using a semi-automatic pipeline, out of which 6 are new sources that were previously unknown or unpublished. Less than half of the sources (31) are firmly identified, the largest population consisting of pulsar wind nebulæ followed by supernova remnants and binary systems. Most of the remaining sources are confused (having several possible counterparts) while a significant fraction (12 sources) have no known counterparts at other wavelengths.

Maps and tables from the HPGS were released, for the first time, as FITS file to allow better exploitation by the community ${ }^{2}$.

\subsection{Population Studies}

For the first time, the existence of a large scale catalogue of $\mathrm{TeV}$ sources made population studies on the dominant source classes, PWN [6] and SNRs [7] possible. One major result is that most young and powerful pulsars have a PWN detected in VHE. H.E.S.S. data also exhibit a correlation of TeV surface brightness with pulsar spin-down power $\dot{E}$, likely caused by an increase of extension with decreasing $\dot{E}$, and hence with time, and also by a mild decrease of $\mathrm{TeV}$ gamma-ray luminosity with decreasing $\dot{E}$. This allows a PWN evolution model to be constructed for the first time, from which it is possible to estimate how many of these objects will be visible by CTA. Regarding SNRs, typical ambient density values around shell-type SNRs can be constrained to $n \leq 7 \mathrm{~cm}^{-3}$ and electron-toproton energy fractions above $10 \mathrm{TeV}$ to $\epsilon_{\mathrm{ep}} \leq 5 \times 10^{-3}$. Comparisons of VHE with radio luminosities also provide support for the theory of magnetic field amplification at shell-type SNRs.

\subsection{Resolving sources down to arcmin scale}

The resolution power of current Imaging Atmospheric Cherenkov Telescopes is presently restricted to scales of a few arc-minutes, and is limited first by statistics and second by the uncertainties in the characterisation of the instrument Point Spread Function (PSF). A new paradigm for the simulation of IACTs has been developed during the last years [15], aiming at overcoming the limitations of current approaches, and to more efficiently cover the simulation phase space. Indeed with the advent of hybrid systems consisting of a large number of IACTs with different sizes, types, and camera configurations, the complexity of the interpolation and the size of the phase space becomes increasingly prohibitive.

In the Run-Wise simulation (RWS) paradigm, in contrast with traditional approach using a fixed grid of simulation parameters, the actual observation conditions as well as the individual telescope configurations of each observation run of a given data set, are taken into account. These run-wise simulations exhibit considerably reduced systematic uncertainties compared to the existing approach. They are also more computationally efficient and simple and therefore opens new physics cases. In particular, the PSF is much better understood and reproduced with the RWS simulation, allowing for the first time the study of the extension of sources down to arc-minute scale, well below the actual PSF size.

Using the RWS simulation scheme,the extension of the Crab nebula at $\mathrm{TeV}$ gamma-ray energies was resolved for the first time [16], with a width of 52" assuming a Gaussian source shape (Fig. 2). The H.E.S.S. extension covers a good fraction of the optical nebula and is significantly larger than the one measured with Chandra. This result can be explained by the radiative cooling of electrons, the

\footnotetext{
${ }^{2}$ These files can be downloaded from https://www.mpi-hd.mpg.de/hfm/HESS/hgps/
} 

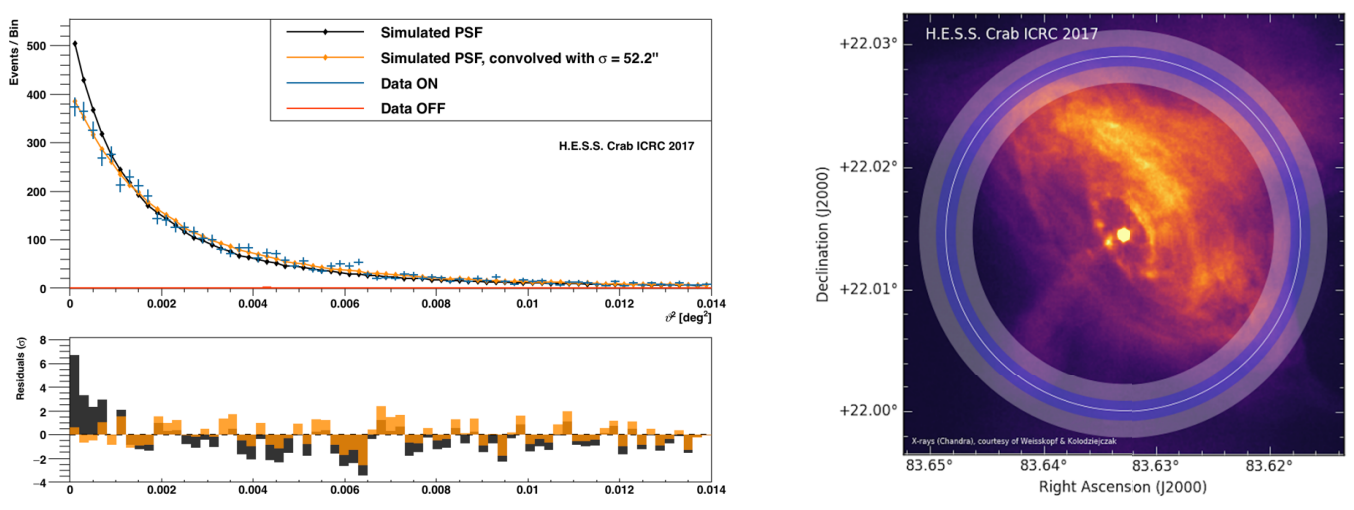

Figure 2. Left: $\theta^{2}$ histogram of $\gamma$-like events from the Crab nebula (ON) and control regions (OFF). Simulated PSF and the PSF convoluted with the best-fit Gaussian are shown as well. Right: Chandra X-ray image of the Crab nebula. The H.E.S.S. extension is shown as solid white circle overlaid on top of shaded annulus indicating the statistical and systematic uncertainties of our measurement. From [16].

size of the nebula decreasing with increasing energies, as measured in other PWNs [e.g. 17], coupled with the fact that the energies of electrons producing the $\mathrm{TeV}$ gamma rays are well below those of electrons emitting the hard X-rays measured by Chandra [e.g. 18].

\section{Extragalactic Observations}

\subsection{Rise of FSRQs}

The advent of the fifth H.E.S.S. telescope, with a threshold of $\sim 20 \mathrm{GeV}$ at zenith, opened up a whole new lower-energy window from which extragalactic observations benefited the most. In particular, several bright FSRQ AGN have been detected recently during outburst episodes: PKS 0736+017 $(z \approx 0.19)$ in February 2015, PKS $1510-089(z \approx 0.36)$ in May 2016 , and 3C $279(z \approx 0.54)$ in July 2015 [19].

The detection of VHE emission from FSRQs during their outbursting episodes was unexpected due to the presence of the broad line region (BLR), an intense radiation field in the vicinity of the AGN, which acts as a barrier to the escape of VHE emission from within it. The detection of VHE emission from these sources can therefore be used to place strong constraints on the position of the emission site with respect to the BLR location. With gamma-ray emission beyond $200 \mathrm{GeV}$ detected from each of these systems during their outbursts, the emission site is found to be constrained to sit at a distance beyond $r_{\mathrm{BLR}} \approx 10^{17} \mathrm{~cm}\left(L_{\mathrm{disk}} / 10^{45} \mathrm{ergs}^{-1}\right)^{1 / 2}$, where $=L_{\mathrm{disk}}$ is the thermal luminosity of the accretion disk [20].

\subsection{Multi-Wavelength, Multi-Messenger astronomy}

Due to its very large effective area and low threshold, H.E.S.S.-II has a competitive advantage over space instruments such as Fermi-LAT for transient phenomena in the overlapping energy range. As speed of reaction is critical for GRBs and other transient phenomena, a rapid repointing system for the telescopes has been implemented [21], which has been designed to respond as fast as possible, 
without human intervention, to targets of opportunity (ToOs). The average overall response time has been reduced to a timescale of $\sim$ few minutes, half of the sky being reachable in less than $60 \mathrm{~s}$.

An example of the recent improvement in response time to neutrino alerts is the follow-up observations of the ANTARES neutrino event on the 30th January 2017. These observations took placed only 32 seconds after the reconstructed neutrino event occurred. Although searches for a gamma-ray counterpart within this data set were unsuccessful, the success of the automatic response system has already proved itself through this considerable reduction in the follow-up delay time to neutrino event alerts. The same applies to gravitational waves, where automatic scheduling is done for events occurring during the night. In the case of the neutron star merger GW170817, the H.E.S.S. telescopes were on target $5.3 \mathrm{~h}$ after the neutron star merger [1, 2], but less than $5 \mathrm{~min}$ after the revised LIGOVIRGO alert was distributed and a handful of seconds after the start of the astronomical night. These 5 min latency was used to implement an observation strategy based on the probability density region obtained by weighting the three-dimensional GW map with galaxies from the GLADE catalog.

\section{Electron spectrum}

The spectrum of high energy electrons, up to $\sim 1 \mathrm{TeV}$ is traditionally measured using space based multi-purpose instruments such as AMS [22] or Fermi-LAT [23], due to much better hadronic rejection than IACTs. Dedicated space based instruments such as CALET or DAMPE are planning to extend the measurement of the electron spectrum up to $\sim 10 \mathrm{TeV}$.

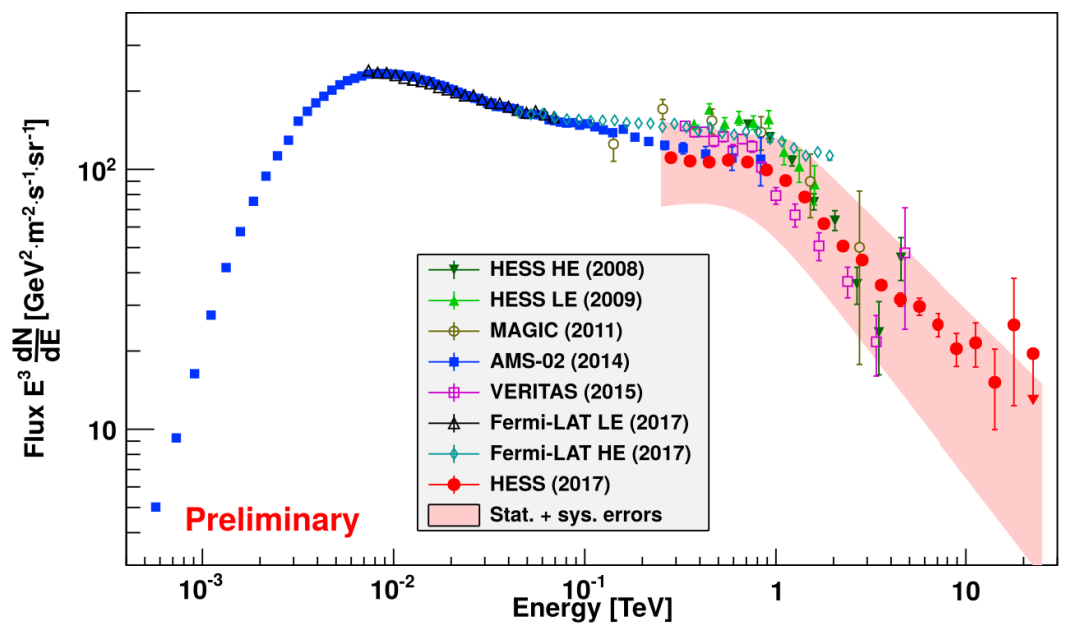

Figure 3. Cosmic-ray electrons energy spectrum measured with H.E.S.S. in 2017 (red dots) compared to previous measurements from various experiments.

In contrast, ground-based Cherenkov telescopes, thanks to their very large effective areas, are able to access the highest energies where the flux becomes very low. More than 9 years after the first electron spectrum measurement with H.E.S.S.[24] up to $5 \mathrm{TeV}$, subsequent observations have increased fourfold the amount of available data. In addition, analysis techniques have improved significantly, leading to a much better suppression of the background of cosmic-ray nuclei. These improvements 
allow a measurement of cosmic-ray electrons up to energies of $\sim 20 \mathrm{TeV}$, presented for the first time at the $35^{\text {th }}$ ICRC, Busan, South Korea (Fig. 3). The measured electron spectrum can be described by two different regimes: up to $\sim 1 \mathrm{TeV}$, the spectrum appears quite regular with a constant slope, and becomes steeper above this energy.

This break in the spectral slope most probably signals the transition between a regime where a large number of sources contribute to the spectrum, to a regime where only the few, closest sources to the Earth are able to contribute. The very high energies reached in this measurement allow models of nearby sources of cosmic-ray electrons in which one source is very prominent to be tested. Such models are often invoked as a possible explanation for the excess of positrons measured by some experiments such as Pamela and AMS.

\section{Conclusions \& Perspectives}

The results presented here represents only a small fraction of the recent highlights from H.E.S.S. Other results were presented at the conference, including in particular - but not only - the discovery of new binary system in the Large Magellanic Cloud, first indications of $\gamma$-ray emission extending beyond the shell of a SNR, a detailed view of the Galactic Center, and evidence for VHE emission from pulsars. After more than 15 years of operation, the H.E.S.S. array is fully refurbished and ready to exploit the opening window of Multi-wavelength, Multi-messenger astronomy before the CTA era.

\section{References}

[1] B.P. Abbott, et al. (LIGO-VIRGO Scientific Collaboration), ApJL 848, L12 (2017)

[2] H. Abdalla, et al. (H.E.S.S. Collaboration), ApJL 850, L22 (2017)

[3] M.G. Aartsen, et al. (IceCube Collaboration), Science 361, eaat1378 (2018)

[4] H. Abdalla, et al. (H.E.S.S. Collaboration), A\&A 612, E1 (2018)

[5] H. Abdalla, et al. (H.E.S.S. Collaboration), A\&A 612, A1 (2018)

[6] H. Abdalla, et al. (H.E.S.S. Collaboration), A\&A 612, A2 (2018)

[7] H. Abdalla, et al. (H.E.S.S. Collaboration), A\&A 612, A3 (2018)

[8] H. Abdalla, et al. (H.E.S.S. Collaboration), A\&A 612, A6 (2018)

[9] H. Abdalla, et al. (H.E.S.S. Collaboration), A\&A 612, A9 (2018)

[10] F. Aharonian, et al (H.E.S.S. Collaboration), Science 307, 1938 (2005)

[11] F. Aharonian, et al (H.E.S.S. Collaboration), ApJ 636, 777 (2006)

[12] F.A. Aharonian, et al., A\&A 395, 803 (2002)

[13] A. Weinstein, et al. (VERITAS Collaboration), ArXiv e-prints (2009), 0912 . 4492

[14] A. Popkow (VERITAS Collaboration), PoS(ICRC) p. 750 (2015)

[15] M. Holler, et al., PoS(ICRC2017) 35, 755 (2017)

[16] M. Holler, et al. (H.E.S.S. Collaboration), PoS(ICRC2017) (2017)

[17] F. Aharonian, et al. (H.E.S.S. Collaboration), A\&A 460, 365 (2006)

[18] A.M. Atoyan, F.A. Aharonian, MNRAS278, 525 (1996)

[19] A.M. Taylor, et al. (H.E.S.S. Collaboration), PoS(ICRC2017) (2017)

[20] G. Ghisellini, F. Tavecchio, MNRAS397, 985 (2009), 0902.0793

[21] C. Hoischen, et al. (H.E.S.S. Collaboration), PoS(ICRC2017) 35, 636 (2017)

[22] M. Aguilar, et al. (AMS Collaboration), Phys. Rev. Lett. 113, 221102 (2014)

[23] S. Abdollahi, et al. (The Fermi-LAT Collaboration), Phys. Rev. D 95, 082007 (2017)

[24] F. Aharonian, et al. (H.E.S.S. Collaboration), Phys. Rev. Lett. 101, 261104 (2008) 